\title{
LIE GROUPS AND PRODUCTS OF SPHERES ${ }^{1}$
}

\author{
P. G. KUMPEL, JR.
}

1. Introduction. In this note, we discuss a problem which was suggested by Serre [12]. Throughout the paper, $G$ denotes a compact, connected, simply connected, simple Lie group. It is a well-known result of Hopf that the real cohomology of $G$ is isomorphic to that of a space $X(G)$, which is a product of odd dimensional spheres:

$$
X(G)=S^{n_{1}} \times \cdots \times S^{n_{l}}, \quad l=\operatorname{rank} G, \quad \sum_{i=1}^{l} n_{i}=\operatorname{dim} G=n .
$$

Serre defines a prime $p$ to be regular for $G$ if there exists a map $f: X(G) \rightarrow G$ such that $f_{*}: H_{i}\left(X(G) ; Z_{p}\right) \rightarrow H_{i}\left(G ; Z_{p}\right)$ is an isomorphism for $i \geqq 0$. In the same paper [12], it is shown that

Theorem 1.1 (Serre). If $p \geqq(\operatorname{dim} G / \operatorname{rank} G)-1$, then $p$ is regular for $G$. If, in addition, $G$ is a classical group, the inequality is necessary for the regularity of $p$.

We prove here the necessity of the inequality $p \geqq(\operatorname{dim} G / \operatorname{rank} G)$ -1 for regularity of $p$ for exceptional Lie groups $G$ and thereby obtain

Theorem 1.2. A prime $p$ is regular for $G$ if and only if $p \geqq(\operatorname{dim} G / \operatorname{rank} G)-1$.

The proof of 1.2, which uses the classification of compact, simple Lie groups, depends heavily on a method of Clark [6] for finding nonzero Steenrod reduced powers.

In his paper [3], Borel calls attention to Serre's work on the problem of comparing $G$ and $X(G)$. He, in fact, states that the above inequality is not necessary in the case of $G$ exceptional because of a result of Toda stating that $\pi_{10}\left(G_{2}\right)=Z_{3}$. This error was later corrected by Bott and Samelson [5]. In $\$ 3$, we give a simple proof of the BottSamelson result that $\pi_{10}\left(G_{2}\right)=0$ using some calculations of Kervaire [10] and a result stated by Harris [7], the proof of which is contained in a forthcoming paper of this author [11].

The author is indebted to Professor Allan Clark for calling to his attention the methods used here, and to Professor Bruno Harris for his advice during the preparation of the paper. This result is con-

Received by the editors November 6, 1964.

1 This research was supported in part by NSF Grant 24026. 
tained in the author's doctoral dissertation and was presented to the American Mathematical Society in February 1964 (Abstract 609-7, Notices Amer. Math. Soc. 11 (1964), 205-207).

2. Proof of Theorem 1.2. In view of Serre's result (1.1), it suffices to show that for exceptional $G$, if $p<(\operatorname{dim} G / \operatorname{rank} G)-1$, then $p$ is irregular for $G$.

Since spheres have no torsion, if $G$ has $p$-torsion, then $p$ is irregular for $G$. Using the definition of regularity, together with the J. H. C. Whitehead theorem (see $[12$, p. 276]), we find that if $p$ is regular for $G$, then the $p$-primary component of $\pi_{j}(G)$ is isomorphic with the direct sum of the $p$-primary components of $\pi_{j}\left(S^{n_{i}}\right), i=1, \cdots, l$. Also, using the naturality of the reduced powers of Steenrod, if there is a nonzero power $P_{p}^{\natural}: H^{i}\left(G ; Z_{p}\right) \rightarrow H^{i+2 i(p-1)}\left(G ; Z_{p}\right)$ then $p$ is irregular for $G$; for the reduced powers are trivial for products of spheres. Hence we may state the

LEMMA 2.1. Each of the following implies that $p$ is irregular for $G$ : (a) $G$ has p-torsion, (b) $\pi_{j}(G)$ and $\bigoplus_{i=1}^{l} \pi_{j}\left(S^{n_{i}}\right)$ do not have isomorphic p-primary components, (c) there is a nonzero $P_{p}^{t}: H^{i}\left(G ; Z_{p}\right)$ $\rightarrow H^{j+2 i(p-1)}\left(G ; Z_{p}\right)$ for some $i \geqq 1$.

In table (2.2), we record for each exceptional group $G$, those primes $p$ for which $G$ has $p$-torsion (see Borel [2]), together with the numbers $\operatorname{dim} G,(\operatorname{dim} G / \operatorname{rank} G)-1$, and the sequences $\left(n_{1}, \cdots, n_{l}\right)$ of dimensions of spheres in $X(G)$ (see Borel and Chevalley [4]).

We proceed to list, for each exceptional group $G$, those primes $p<(\operatorname{dim} G / \operatorname{rank} G)-1$, such that $G$ has no $p$-torsion, $p \neq 3$ if $G=G_{2}$. For such $p$ we note the pairs $\left(m_{j}, m_{k}\right)$ satisfying the hypotheses of Clark's theorem (2.3). We then record the corresponding nonzero $P_{p}^{1}$.

(2.2)

\begin{tabular}{|c|c|c|l|l|}
\hline$G$ & $\operatorname{dim} G$ & $\left(\frac{\operatorname{dim} G}{\operatorname{rank} G}\right)-1$ & $p$-torsion & \multicolumn{1}{|c|}{$\left(n_{1}, \cdots, n_{l}\right)$} \\
\hline$G_{2}$ & 14 & 6 & 2 & $(3,11)$ \\
$F_{4}$ & 52 & 12 & 2,3 & $(3,11,15,23)$ \\
$E_{8}$ & 78 & 12 & 2,3 & $(3,9,11,15,17,23)$ \\
$E_{7}$ & 133 & 18 & 2,3 & $(3,11,15,19,23,27,35)$ \\
$E_{8}$ & 248 & 30 & $2,3,5$ & $(3,15,23,27,35,39,47,59)$ \\
\hline
\end{tabular}

Bott and Samelson [5] have shown that $\pi_{10}\left(G_{2}\right)$ has 3-primary 
component zero. Since $\pi_{10}\left(S^{2} \times S^{11}\right)_{3}=Z_{3}$ (see [12, p. 285]), by (2.1b), 3 is irregular for $G_{2}$. (See also Proposition 3.1.) From the table (2.2) of $p$-torsion, and (2.1a), together with the preceding remark, it follows that 2 and 3 are irregular for all exceptional groups, and that 5 is irregular for $E_{8}$.

Now we show that for exceptional $G$, if $p<(\operatorname{dim} G / \operatorname{rank} G)-1$, $p \neq 2,3$ and $p \neq 5$ if $G=E_{8}$, there exists a nonzero reduced power $P_{p}^{t}: H^{i}\left(G ; Z_{p}\right) \rightarrow H^{i+2 i(p-1)}\left(G ; Z_{p}\right)$. This result will complete the proof of (1.2) using (2.1c).

The following is a rewording of a result due to Clark [6]. We state and prove it in a form which is convenient for our purposes.

Theorem 2.3 (Clark). Let $G$ be as in (1.2). Let $H^{*}(G ; R)$ be an exterior algebra on generators $x_{n_{i}}, i=1, \cdots, l, \operatorname{deg} x_{n_{i}}=n_{i}=2 m_{i}-1$. If $p$ is prime and $G$ has no $p$-torsion and if there exists $k, 1 \leqq k \leqq l$, such that (i) $m_{k} \neq \equiv(\bmod p)$, (ii) $m_{k}>p$, (iii) the set $\left\{m_{1}, \cdots, m_{l}\right\}$ contains exactly one element $m_{j}$ such that $m_{j} \equiv(1-p) \bmod m_{k}$, and $m_{j}<m_{k}$, then $P_{0}^{1} x_{2 m_{j-1}}=x_{2 m_{k}-1}$.

Proof of (2.3). Since $G$ has no $p$-torsion, the classifying space $B$ of $G$ has the property that $H^{*}\left(B ; Z_{p}\right)$ is a polynomial algebra on generators $y_{2 m_{i}}, i=1, \cdots, l$, which suspend to the generators $x_{2 m_{i}-1}$. Let $m_{k}$ be as in the statement of the theorem. Let $I$ be the ideal of $H^{*}\left(B ; Z_{p}\right)$ generated by the $y_{2 m_{i}}, i \neq k$. Then either $P_{p}^{1}(I) \subset I$ or $P_{p}^{1}(I) \circlearrowleft I$.

We shall now see that the first of these is impossible. Since $m_{k}>p$, we may write $m_{k}=a+b p, b>0,0<a<p$. As a special case of the Adem relations, [1], $P_{p}^{a} P_{p}^{b p} y_{2 m_{k}}=P_{p}^{m_{k}} y_{2 m_{k}}=y_{2 m_{k}}^{p}$. Since $P_{p}^{1}(I) \subset I$, we have $P_{p}^{a}(I) \subset I$, for $a ! P_{p}^{a}=\left(P_{p}^{1}\right)^{a}$, and $a ! \neq 0(\bmod p)$. Hence, if $P_{p}^{b p} y_{2 m_{k}} \in I$, we have $y_{2 m_{k}}^{p} \in I$, which is a contradiction. Therefore $P_{p}^{p o p} y_{2 m_{k}} \in I$. Thus, $P_{p}^{o p} y_{2 m_{k}}=q y_{2 m_{k}}^{r}+s, q \neq 0, q \in Z_{p}, s \in I$. Taking degrees we get $2 m_{k}+2 b p(p-1)=2 r m_{k}$. Hence $m_{k}(r-1)=b p(p-1)$. Since $m_{k}>b p, r<p$, and since $p$ is prime, and $r-1$ divides $b p(p-1)$, we see that $r-1$ divides $b(p-1)$. Hence $m_{k} \equiv 0(\bmod p)$, a contradiction. Thus $P_{p}^{1}(I) \nsubseteq I$.

If all generators of $I$ were mapped into $I$ by $P_{p}^{1}$, we would have $P_{p}^{1}(I) \subset I$. For an element of $I$ can be written as a sum of elements of the form $u_{i} y_{2 m_{i}}, u_{i} \in H^{*}\left(B ; Z_{p}\right)$. But $P_{p}^{1}$ preserves sums and $P_{p}^{1}\left(u_{i} y_{2 m_{i}}\right)$ $=P_{p}^{1}\left(u_{i}\right) y_{2 m_{i}}+\epsilon u_{i} P_{p}^{1} y_{2 m_{i}}, \epsilon= \pm 1$, which is in $I$ if $P_{p}^{1}\left(y_{2 m_{i}}\right) \in I$. Therefore $P_{p}^{1}\left(y_{2 m_{i}}\right) \notin I$ for some $i \neq k$, that is $P_{p}^{1} y_{2 m_{i}}=q y_{2 m_{k}}^{r}+s, q \neq 0, s \in I$.

Taking degrees, we get $2 m_{i}+2(p-1)=(r)\left(2 m_{k}\right)$, i.e., $m_{i}=(1-p)$ $+r m_{k}$. Hence $m_{i} \equiv(1-p) \bmod m_{k}$. By hypothesis, there is only one 
such $m_{i}$, called $m_{j}$. Moreover, $m_{j}<m_{k}$. We show that $r=1$. If $r>1$, then $(r-1) m_{k}>p$, hence $(r-1) m_{k}+m_{k}-m_{j}>p$, or $r m_{k}-m_{j}>p>p$ -1 , i.e., $m_{j}<(1-p)+r m_{k}$ contrary to the fact that $m_{j}=(1-p)$ $+r m_{k}$. Hence $m_{j}=(1-p)+m_{k}$, and $P_{p}^{1} y_{2 m_{j}}=q y_{2 m_{k}}+s$. Since $s$ is decomposable, it is mapped into zero by the suspension, and by the naturality we obtain $P_{p}^{1} x_{2 m_{j-1}}=x_{2 m_{k-1}}$. We may assume $q=1$ by suitably choosing the generators $x_{i}$. This proves (2.3).

We now apply (2.3) to complete the proof of (1.2). First note from (2.2) that for $G_{2}, F_{4}, E_{6}, E_{7}, E_{8}$, the sequences $\left(m_{1}, \cdots, m_{l}\right), m_{i}$ $=\left(n_{i}+1\right) / 2$ are, respectively, $(2.6),(2,6,8,12),(2,5,6,8,9,12)$, $(2,6,8,10,12,14,18)$, and $(2,8,12,14,18,20,24,30)$.

\begin{tabular}{|c|c|c|c|}
\hline$G$ & $p$ & $\left(m_{j}, m_{k}\right)$ & nonzero $P_{p}^{1}$ \\
\hline$G_{2}$ & 5 & $(2,6)$ & $P_{8}^{1} x_{3}=x_{11}$ \\
\hline \multirow[t]{4}{*}{$F_{4}$} & 5 & $(8,12)$ & $P_{5}^{1} x_{15}=x_{23}$ \\
\hline & 7 & $(2,8)$ & $P_{7}^{1} x_{3}=x_{15}$ \\
\hline & & $(6,12)$ & $P_{7}^{1} x_{11}=x_{28}$ \\
\hline & 11 & $(2,12)$ & $P_{11}^{1} x_{8}=x_{23}$ \\
\hline \multirow[t]{5}{*}{$E_{6}$} & 5 & $(5,9)$ & $P_{5}^{1} x_{9}=x_{17}$ \\
\hline & & $(8,12)$ & $P_{5}^{1} x_{15}=x_{23}$ \\
\hline & 7 & $(2,8)$ & $P_{7}^{1} x_{3}=x_{15}$ \\
\hline & & $(6,12)$ & $P_{7}^{1} x_{11}=x_{23}$ \\
\hline & 11 & $(2,12)$ & $P_{11}^{1} x_{3}=x_{23}$ \\
\hline \multirow[t]{8}{*}{$E_{7}$} & 5 & $(8,12)$ & $P_{8}^{1} x_{15}=x_{28}$ \\
\hline & & $(10,14)$ & $P_{b}^{1} x_{10}=x_{27}$ \\
\hline & & $(14,18)$ & $P_{5}^{1} x_{27}=x_{35}$ \\
\hline & 7 & $(12,18)$ & $P_{7}^{1} x_{23}=x_{35}$ \\
\hline & 11 & $(8,18)$ & $P_{11}^{1} x_{15}=x_{36}$ \\
\hline & 13 & $(2,14)$ & $P_{13}^{1} x_{3}=x_{27}$ \\
\hline & & $(6,18)$ & $P_{13}^{1} x_{11}=x_{35}$ \\
\hline & 17 & $(2,18)$ & $P_{17 x_{3}}^{1}=x_{25}$ \\
\hline
\end{tabular}




\begin{tabular}{|c|c|c|c|}
\hline$G$ & $p$ & $\left(m_{j}, m_{k}\right)$ & nonzero $P_{p}^{1}$ \\
\hline \multirow[t]{15}{*}{$E_{8}$} & 7 & $(14,20)$ & $P_{7}^{1} x_{27}=x_{39}$ \\
\hline & & $(18,24)$ & $P_{7}^{1} x_{35}=x_{47}$ \\
\hline & & $(24,30)$ & $P_{7}^{1} x_{47}=x_{69}$ \\
\hline & 11 & $(8,18)$ & $P_{11}^{1} x_{15}=x_{35}$ \\
\hline & & $(14,24)$ & $P_{11}^{1} x_{27}=x_{47}$ \\
\hline & & $(20,30)$ & $P_{11}^{1} x_{39}=x_{59}$ \\
\hline & 13 & $(8,20)$ & $P_{13}^{1} x_{15}=x_{39}$ \\
\hline & & $(12,24)$ & $P_{13}^{1} x_{23}=x_{47}$ \\
\hline & & $(18,30)$ & $P_{13}^{1} x_{35}=x_{59}$ \\
\hline & 17 & $(8,24)$ & $P_{17}^{1} x_{15}=x_{47}$ \\
\hline & & $(14,30)$ & $P_{17}^{1} x_{27}=x_{59}$ \\
\hline & 19 & $(2,20)$ & $P_{19}^{1} x_{3}=x_{39}$ \\
\hline & & $(12,30)$ & $P_{19}^{1} x_{23}=x_{59}$ \\
\hline & 23 & $(2,24)$ & $P_{23}^{1} x_{3}=x_{47}$ \\
\hline & & $(8,30)$ & $P_{23}^{1} x_{15}=x_{59}$ \\
\hline & 29 & $(2,30)$ & $P_{29}^{1} x_{3}=x_{59}$ \\
\hline
\end{tabular}

In each case, the fact that $\left(m_{j}, m_{k}\right)$ satisfies the hypotheses of Theorem 2.3 is easily checked. Thus (1.2) is established.

3. Computation of $\pi_{10}\left(G_{2}\right)$. Finally we prove the following proposition, whose significance was noted in the introduction.

Proposition 3.1. $\pi_{10}\left(G_{2}\right)=0$.

Proof. In Harris's paper [7], it is stated that the homotopy exact sequence of the fibration $G_{2} \rightarrow \operatorname{Spin} 8 \rightarrow \operatorname{Spin} / G_{2}$ is split when tensored with $Q_{3}$, the ring of rational numbers whose denominators are powers of 3. The splitting is given by the map

$$
q: \text { Spin 8/G } \rightarrow \text { Spin 8, } q\left(\tau G_{2}\right)=\tau \sigma(\tau)^{-1}, \quad \tau \in \operatorname{Spin} 8,
$$

$\sigma$ the automorphism of order 3 of Spin 8 having $G_{2}$ as fixed point set. 
(A description of the inclusion $G_{2} \subset$ Spin 8 and a proof of this splitting are contained in a forthcoming paper of the author [11].)

Let $p$ : Spin $8 \rightarrow$ Spin $8 / G_{2}$ be the projection. It is known that Spin $8 / G_{2}$ is homeomorphic with $S^{7} \times S^{7}$ (see [9, II, p. 93]). In the proof of the above splitting, it was shown that if we identify

then

$$
\pi_{j}\left(\text { Spin } 8 / G_{2}\right) \approx \pi_{j}\left(S^{7}\right) \oplus \pi_{j}\left(S^{7}\right),
$$

$$
p_{*} q_{*}(\alpha, \beta)=(\alpha+\beta, 2 \beta-\alpha), \quad \alpha, \beta \in \pi_{j}\left(S^{7}\right) .
$$

We record the following results on homotopy groups of spheres and orthogonal groups:

$$
\begin{aligned}
\pi_{n+4}\left(S^{n}\right) & =0, \quad n \geqq 6, \\
\pi_{n+3}\left(S^{n}\right) & =Z_{24}, \quad n \geqq 5, \\
\pi_{8++2}(S O(8 s)) & =Z_{24} \oplus Z_{8}, \quad s \geqq 1 .
\end{aligned}
$$

The first two can be found in $\mathrm{Hu}$ [8, pp. 329-331], and the last is due to Kervaire [10].

Take $n=7, s=1$, and observe that the exact homotopy sequence of the above fibering

$$
\cdots \rightarrow \pi_{11}\left(S^{7} \times S^{7}\right) \stackrel{\partial}{\rightarrow} \pi_{10}\left(G_{2}\right) \stackrel{j^{*}}{\rightarrow} \pi_{10}(\operatorname{Spin} 8) \stackrel{p^{*}}{\rightarrow} \pi_{10}\left(S^{7} \times S^{7}\right) \rightarrow \cdots
$$

reduces to the exact sequence

$$
0 \stackrel{\partial}{\rightarrow} \pi_{10}\left(G_{2}\right) \stackrel{j^{*}}{\rightarrow} Z_{24} \oplus Z_{8} \stackrel{p^{*}}{\rightarrow} Z_{24} \oplus Z_{24}
$$

since $\pi_{n}(\operatorname{Spin} 8) \approx \pi_{n}(S O(8)), n \geqq 2$.

If $\pi_{10}\left(G_{2}\right)$ has an element $x$ of order 3 , then $j_{*}(x) \neq 0$ and has order 3 , therefore generates the 3-component of $\pi_{10}(\operatorname{Spin} 8) \approx Z_{24} \oplus Z_{8}$ $\approx Z_{3} \oplus Z_{8} \oplus Z_{8}$. Since $p_{*} j_{*}=0$, all elements of order 3 are in ker $p_{*}$. Let $\alpha \in \pi_{10}\left(S^{7}\right) \approx Z_{24}$ be an element of order 3, $\alpha \neq 0$. Then $q_{*}(\alpha, 0)$ $\in \pi_{10}(\operatorname{Spin} 8)$ has order 3 or is zero. In any case, $0=p_{*} q_{*}(\alpha, 0)$ $=(\alpha,-\alpha)$, hence $\alpha=0$, a contradiction. Hence $\pi_{10}\left(G_{2}\right)_{3}=0$.

Finally, from Serre [12, Corollary 2, p. 289], we know that $\pi_{10}\left(G_{2}\right)$ is finite. From the fact that the homotopy sequence of $G_{2} \rightarrow$ Spin $8 \rightarrow$ Spin $8 / G_{2}$ is split upon tensoring with $Q_{3}$, we obtain

$$
\left(\pi_{10}\left(G_{2}\right) \oplus \pi_{10}\left(S^{7} \times S^{7}\right)\right) \otimes Q_{3} \approx \pi_{10}(\text { Spin } 8) \otimes Q_{3} .
$$

Therefore

$$
\left(\pi_{10}\left(G_{2}\right) \otimes Q_{3}\right) \oplus Z_{8} \oplus Z_{8} \approx Z_{8} \oplus Z_{8}
$$

Hence $\pi_{10}\left(G_{2}\right) \otimes Q_{3}=0$, i.e., $\pi_{10}\left(G_{2}\right)$ has no elements of finite order $\neq 3$. We have seen that $\pi_{10}\left(G_{2}\right)_{3}=0$, hence 3.1 is proved. 


\section{REFERENCES}

1. J. Adem, The relations on Steenrod reduced powers of cohomology classes, Algebraic Geometry and Topology, pp. 191-238, Princeton Univ. Press, Princeton, N. J., 1957.

2. A. Borel, Sous-groupes commutatifs et torsion des groupes de Lie compacts connexes, Tohoku Math. J. 13 (1961), 216-240.

3. - Topology of Lie groups and characteristic classes, Bull. Amer. Math. Soc. 61 (1955), 273-342.

4. A. Borel and C. Chevalley, The Betti numbers of the exceptional groups, Mem. Amer. Math. Soc. 14 (1955), 1-9.

5. R. Bott and H. Samelson, Application of the theory of Morse to symmetric spaces, Amer. J. Math. 53 (1958), 964-1029.

6. A. Clark, On $\pi_{3}$ of finite-dimensional H-spaces, Ann. of Math. 78 (1963), 193196.

7. B. Harris, Suspensions and characteristic maps for symmetric spaces, Ann. of Math. 76 (1962), 295-305.

8. S.-T. Hu, Homotopy theory, Academic Press, New York, 1959.

9. N. Jacobson, Some groups of transformations defined by Jordan algebras. I, II, III, J. Reine Angew. Math. 201 (1959), 178-195, ibid. 204 (1960), 74-98, ibid. 207 (1961), 61-85.

10. M. Kervaire, Some non-stable homotopy groups of Lie groups, Illinois J. Math. 4 (1960), 161-169.

11. P. G. Kumpel, Jr., On the homotopy groups of the exceptional Lie groups, Trans. Amer. Math. Soc. 120 (1965), 481-498.

12. J.-P. Serre, Groupes d'homotopie et classes de groupes abeliens, Ann. of Math. 58 (1953), 258-294.

\section{BROWN UNIVERSITY AND}

State University of New York at Stony Brook 